\title{
UM ESTUDO SOBRE LETRAMENTO \\ DIGITAL PARA IDOSOS COM O APOIO DE UM APLICATIVO MÓVEL PERSONALIZÁVEL
}

\author{
Isabela Zaine ${ }^{1}$ \\ Kamila Rios da Hora Rodrigues² \\ Caio César Viel ${ }^{3}$ \\ Bruna Carolina Rodrigues da Cunha ${ }^{4}$ \\ Samila Sathler Tavares Batistoni ${ }^{5}$ \\ Maria da Graça Campos Pimentel ${ }^{6}$ \\ Meire Cachioni7
}

1 Graduada em Psicologia. Doutora em Psicologia. Psicóloga clínica. E-mail: isabela.zaine@gmail.com.

2 Bacharel em Ciência da Computação. Doutora em Ciências da Computação. Professora Doutora da Universidade de São Paulo (USP), vinculada ao Instituto de Ciências Matemáticas e de Computação. E-mail: kamila.rios@icmc.usp.br.

3 Graduado em Engenharia da Computação. Doutor em Ciências da Computação e Matemática Computacional. Engenheiro de Software do SIDIA Instituto de Ciências e Tecnonogia. E-mail: caio. viel@sidia.com.

4 Bacharel em Ciências da Computação. Doutora em Ciências da Computação e Matemática Computacional. Professora no Instituto Federal de São Paulo (IFSP) campus Capivari. E-mail: bruna. rodrigues@ifsp.edu.br.

5 Graduada em Psicologia. Doutora em Educação. Professora Doutora da Universidade de São Paulo (USP), vinculada à Escola de Artes, Ciências e Humanidades. E-mail: samilabatistoni@usp.br.

6 Bacharel em Ciências da Computação. Doutora em Computação. Professora Titular da Universidade de São Paulo (USP), vinculada ao Instituto de Ciências Matemáticas e de Computação. E-mail: mgp@icmc.usp.br.

7 Graduada em Psicologia. Doutora em Gerontologia. Professora Associada da Universidade de São Paulo (USP), vinculada à Escola de Artes, Ciências e Humanidades. E-mail: mailto:meire@usp.br. 
O uso de sistemas computacionais para cuidados com a saúde de idosos é cada vez mais comum. No campo da educação, no entanto, as investigações enfocam mais em questões técnicas de desenvolvimento ou em adaptação de tecnologias, e não no processo de ensino-aprendizagem e aplicabilidade do uso de dispositivos móveis. Este trabalho buscou estimular, monitorar e avaliar o uso desses dispositivos no ambiente natural por idosos participantes de cursos de alfabetização e letramento digital. Também buscou promover novas metodologias para práticas de alfabetização e letramento digital para idosos. Foi realizado um estudo de viabilidade explorando o uso de um sistema denominado ESPIM, como um novo recurso pedagógico em cursos como este. O ESPIM permite a profissionais de diferentes áreas construírem programas de intervenção para realizar coletas de dados de forma remota com suas populações de interesse. Essa população responde a perguntas programadas pelos profissionais usando um aplicativo móvel. As perguntas planejadas podem ser questões abertas, de múltipla escolha, de escolha única, solicitação do envio de mídias como áudio, vídeo ou imagens. Neste estudo qualitativo, por duas semanas consecutivas, os participantes receberam alertas e instruções, por meio desse aplicativo, para realizar atividades práticas em casa sobre o conteúdo aprendido em sala de aula. Os resultados obtidos descrevem um comportamento positivo dos participantes em termos de engajamento na execução das atividades de casa, e também descrevem feedbacks sobre o aplicativo, tais como facilidades e dificuldades, autonomia no uso e adesão ao recurso tecnológico como forma de apoio a cursos como o supracitado.

palavras-chave

Idosos. Alfabetização e Letramento Digital. Sistema ESPIM. Dispositivos Móveis. Intervenções Remotas. 
a partir de práticas de leituras, releituras de informações e escrita, a fim de utilizar as tecnologias como benefício na vida pessoal e coletiva. A inserção de indivíduos em práticas sociais por meio de uma análise crítica das informações disponibilizadas (MACHADO; LONGHI; BEHAR, 2013), tem por objetivo tornar as pessoas capazes de usufruir dos recursos tecnológicos e de desenvolver a criticidade em sua utilização ao longo da vida.

Neste sentido, dois grupos de pesquisa da Universidade de São Paulo, um do Instituto de Ciências Matemáticas e de Computação da cidade de São Carlos e outro do Programa de Pós-Graduação em Gerontologia da Escola de Artes, Ciências e Humanidades, já ofereciam há algum tempo cursos de alfabetização e letramento digital de idosos em relação ao uso de dispositivos móveis, tais como os smartphones e tablets. As práticas com cada nova turma evidenciaram uma dificuldade no processo de ensino-aprendizagem comum aos dois grupos de pesquisa, por exemplo: a de generalização dos conteúdos aprendidos em sala de aula em relação ao ambiente natural do idoso, isto é, fora da instituição de ensino. Muitas vezes os idosos só conseguiam interagir com algumas funcionalidades dos dispositivos em sala de aula, com a ajuda dos professores e monitores.

Diante do engajamento dos idosos no uso dos dispositivos em domicílio, os grupos de pesquisa adotaram um modelo intitulado ESPIM - Experience Sampling and Programmed Intervention Method. Esse modelo é apoiado por um sistema computacional de mesmo nome, desenvolvido por pesquisadores do Instituto de Ciências Matemáticas e de Computação (ZAINE et al., 2016; VIEL et al., 2017; RODRIGUES et al., 2018) e que oferece alternativas para a realização de coleta de dados e de intervenções programadas por especialistas de diferentes áreas de domínio, tais como a saúde e a educação (RODRIGUES et al., 2018; ZAINE et al., 2016).

O sistema ESPIM pode ser utilizado por profissionais da Educação para a coleta de dados da sua população de interesse e/ou no estímulo ao uso e engajamento com tecnologias móveis. Neste sentido, este trabalho relata o uso de tal sistema como "ajudante digital" de idosos que frequentavam as aulas dos cursos de letramento digital oferecidos pela EACH e ICMC. Trata-se de um estudo piloto e qualitativo que forneceu subsídios para a reformulação da metodologia de ensino adotada nos referidos cursos de letramento. A Seção 2 deste artigo descreve o sistema ESPIM, suas principais funcionalidades e componentes. A Seção 3 descreve o método utilizado no estudo. A Seção 4 discute os resultados obtidos. A Seção 5 traz uma discussão sobre os achados e, a Seção 6 aponta as considerações finais. 


\section{Sistema ESPIM}

O ESPIM ${ }^{8}$ disponibiliza duas interfaces de usuário, uma Web para especialistas e outra para dispositivos móveis Android, usada pelas populações de interesse desses especialistas (CUNHA et al., 2018; VIEL et al., 2017). O sistema permite o planejamento e a autoria de intervenções baseadas em dados coletados de maneira explícita e pervasiva, por meio dos sensores físicos do dispositivo. Permite, ainda, a troca de dados entre as diferentes partes interessadas envolvidas no planejamento da coleta de dados e/ou intervenção, como professores e alunos (ZAINE et al., 2016; PIMENTEL et al., 2016). Por meio do uso do ESPIM é possível ampliar o alcance da coleta de dados e intervenções realizadas de maneira remota, no ambiente natural dos participantes de interesse, e sem a presença direta do pesquisador. A concepção do ESPIM foi inspirada em procedimentos e metodologias da Psicologia e Computação Ubíqua, a qual considera que as pessoas fazem uso da tecnologia de maneira natural e, por essa fazer parte de seu ambiente cotidiano, pode ser usada para ajudar a cumprir tarefas do dia a dia (WEISER, 1991). Assim, a tecnologia se torna parte do ambiente natural das pessoas. Smartphones representam bem tal conceito. Por meio desses dispositivos os usuários podem ser imediatamente sinalizados para realizar relatos de experiência de diversas formas (e.g. texto, vídeo, fotografia) e o envio de dados pode ser feito imediatamente ao pesquisador. Além disso, esses dispositivos podem ser usados como meio de implementação de planos de intervenções programadas (ZAINE et al., 2016).

Uma das influências dentro do campo da Psicologia foi o Método de Amostragem de experiências, Experience Sampling Method - ESM (CSIKSZENTMIHALYI; LARSON, 1987), que operacionaliza maneiras de coletar informações sistêmicas sobre a experiência das pessoas em ambiente natural. No entanto, há momentos em que, além de coletar dados, existe o interesse de intervir na situação de alguma maneira, como forma de provocar alguma alteração de comportamento. Assim, uma segunda influência na concepção do ESPIM vem do campo da Análise do Comportamento, mais especificamente da área de programação de ensino. Os princípios, procedimentos e tecnologias produzidos por esse campo são generalizáveis para diferentes contextos, cuja finalidade é instalar ou alterar comportamentos, ou reduzir a probabilidade de ocorrência dos mesmos. No presente estudo, chamamos o resultado de tal programação de ensino de "intervenções programadas".

8 Disponivel em: https://www.espim.com.br. Acesso em: 18 abr. 2019. 
Os componentes de infraestrutura computacional do ESPIM incluem: um aplicativo móvel para a plataforma Android, utilizado pela população de interesse estudada pelos especialistas; um sistema Web, para o planejamento das coletas e intervenções, utilizado por pesquisadores e profissionais e, um Web Service, responsável pelo armazenamento das informações (CUNHA et al., 2018; VIEL et al., 2017).

A interface Web do ESPIM permite a criação de planos individualizados de intervenções programadas, cadastro de participantes e visualização dos resultados enviados ao servidor. Por meio da interface Web, o pesquisador pode programar diferentes maneiras de interação de participantes com o sistema, tanto em termos de recebimento quanto de envio de informações de interesse. Por exemplo, podem ser criados programas interventivos compostos por questões do tipo aberta, múltipla escolha com uma ou mais possibilidades de resposta, mensagens instrucionais/informativas. O fluxo entre uma questão e outra pode ser diferente de acordo com a resposta providenciada. As questões podem ser programadas em formato de texto, áudio ou vídeo e os participantes podem responder a elas usando qualquer um desses formatos. O pesquisador pode enviar um vídeo aos participantes com instruções de como uma atividade deve ser realizada, ou até mesmo um vídeo-modelo de uma atividade específica e solicitar aos participantes que gravem e enviem um vídeo deles mesmos realizando a atividade. Os dados produzidos são, então, enviados automaticamente ao servidor e ficam disponíveis no sistema para o pesquisador.

Na interface Web, o pesquisador também define um intervalo em que o protocolo de intervenção ou de coleta de dados deverá ficar ativo. O sistema também permite que os participantes recebam notificações sonoras sempre que houver alguma solicitação programada no sistema. Para tanto, um gatilho temporal é definido para ser acionado em um horário específico no dispositivo do participante, por exemplo, todos os dias às 11:00, ou todas as segundas, quartas e sextas às 8:00, 14:00 e 19:00, respectivamente. O gatilho temporal serve como um lembrete para os participantes interagirem com o aplicativo. O formato do lembrete também é customizável, podendo ser mais perceptível, como um despertador, ou mais sutil, como uma simples notificação (RODRIGUES et al., 2018).

A Figura 1 ilustra um exemplo de plano de intervenções individualizado criado na interface Web do ESPIM. Nesse exemplo é possível verificar questões dos tipos: múltipla escolha e múltiplas opções, tarefa, questão aberta e mensagem. 
Figura 1 - Exemplo de Plano de Intervenções no ESPIM Web ESPIM Q Q

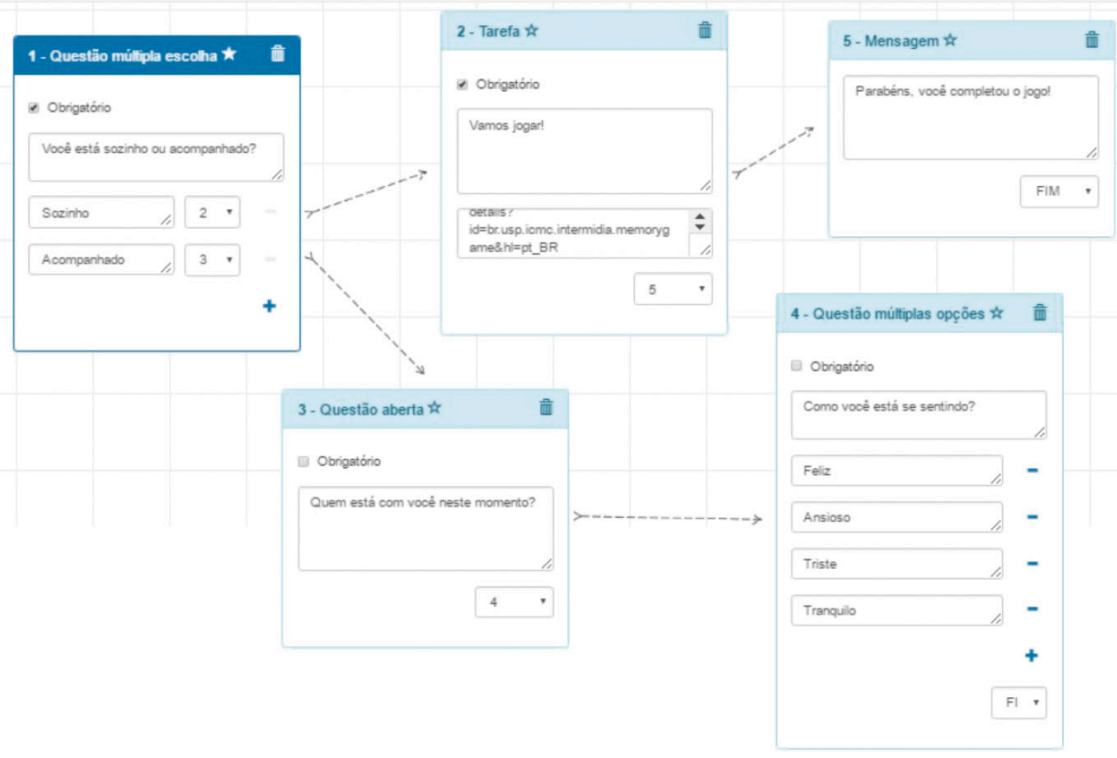

Fonte: Rodrigues et al. (2018, p. 4).

A aplicação móvel do sistema recupera essas informações programadas no sistema Web e gera telas para que o participante possa interagir em seu dispositivo móvel (CUNHA et al., 2018). A Figura 2 ilustra parte das intervenções da Figura 1 disponibilizada no dispositivo móvel do participante.

Figura 2 - Fluxo 2 da intervenção proposta na Figura 1 (se o participante estiver acompanhado: perguntar quem o acompanha e qual o seu humor)

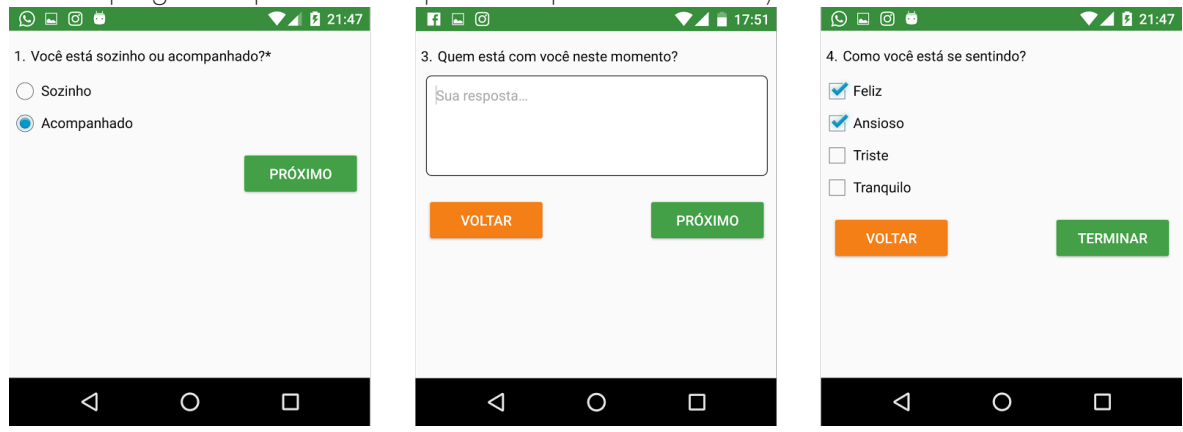

Fonte: Rodrigues et al. (2018, p. 5). 
Baseado nas funcionalidades oferecidas pelo sistema ESPIM, foi planejado um estudo de viabilidade explorando o uso do mesmo como um novo recurso pedagógico em cursos de alfabetização e letramento digital voltados ao público idoso com o foco no ensino sobre o uso de dispositivos móveis. O principal objetivo deste estudo foi o de monitorar o uso de dispositivos móveis pelos idosos, bem como monitorar a realização das atividades remotas, por meio do aplicativo móvel do ESPIM. Um segundo objetivo foi o de promover novas metodologias, procedimentos e práticas pedagógicas para a alfabetização e letramento digital de idosos.

\section{Método}

Participaram do estudo sete idosos matriculados em cursos de extensão universitária oferecidos pelos grupos de pesquisa da USP, EACH (instituição A) e ICMC (instituição B), que visam o letramento digital para o uso de dispositivos móveis. Quatro eram do sexo feminino e três do sexo masculino, com idades entre 57 e 82 anos $(M=69,2)$, conforme Tabela 1.

Tabela 1 - Participantes

\begin{tabular}{lllllll}
\hline P & Sexo & Idade & Escolaridade & Profissão & Dispositivo & Instituição \\
\hline P1 & F & 57 & Superior & Aposentada & Tablet & A \\
P2 & M & 70 & Médio & Aposentado & Tablet & A \\
P3 & F & 74 & Superior & Aposentada & Smartphone & A \\
P4 & M & 82 & Superior & Aposentado & Smartphone & A \\
P5 & F & 62 & Médio & Aposentada & Smartphone & B \\
P6 & F & 65 & Médio & Aposentada & Smartphone & B \\
P7 & M & 75 & Médio & Aposentado & Smartphone & B \\
\hline
\end{tabular}

Fonte: Elaborada pelos autores.

Em relação ao uso de dispositivos móveis, apenas três dos sete participantes já faziam uso dos mesmos antes de se inscreverem nos cursos de práticas com dispositivos móveis. No entanto, na ocasião deste estudo, todos os participantes relataram fazer uso de tais dispositivos diariamente: três participantes relataram usar uma vez ao dia e quatro relataram usar os dispositivos mais de uma vez ao dia. Dentre as funções mais mencionadas, para as quais 
os participantes usavam seus tablets/smartphones, pode-se citar, em ordem de frequência: 1) comunicação, 2) pesquisa na Internet, e 3) compras e envio e recebimento de mídias. A Tabela 2 descreve individualmente as informações sobre o uso dos dispositivos.

Tabela 2 - Dados individuais acerca do uso de tablets/smartphones

\begin{tabular}{llll}
\hline P & $\begin{array}{l}\text { Uso } \\
\text { do dispositivo } \\
\text { antes do curso }\end{array}$ & Frequência atual de uso & Funções usadas no cotidiano \\
\hline P1 & Não & Diariamente: uma vez ao dia & $\begin{array}{l}\text { Comunicação, e-mail, pesquisa } \\
\text { Internet, fotos e vídeos }\end{array}$ \\
P2 & Não & Diariamente: uma vez ao dia & Comunicação \\
P3 & Não & Diariamente: mais de uma & Comunicação, compras, rede \\
P4 & Sim & vez ao dia & sociais \\
P5 & Sim & Diariamente: uma vez ao dia & Comunicação \\
& & vez ao dia & Comunicação, pesquisa Internet \\
P6 & Não & Diariamente: mais de uma & Comunicação \\
& Pez ao dia & Diariamente: mais de uma & Comunicação \\
\hline
\end{tabular}

Fonte: Elaborada pelos autores.

\subsection{Considerações éticas}

Esta pesquisa foi aprovada pelo Comitê de Pesquisa em Seres Humanos da EACH-USP, com protocolo número: 2.171.716. Os termos de consentimento, bem como os questionários de pré e pós-testes foram aplicados seguindo planejamento descrito no projeto do comitê de ética aprovado.

\subsection{Materiais e local}

Os materiais utilizados durante a pesquisa incluíram tablets e smartphones pessoais dos participantes equipados com o sistema operacional Android, a partir da versão 4.4, e com o aplicativo móvel do ESPIM instalado. As atividades foram executadas remotamente nas residências de cada participante. 
Foram usados os seguintes instrumentos para coletar os dados: 1) uma entrevista semiestruturada com 11 questões sobre o engajamento nas atividades em casa, o envolvimento de outras pessoas para ajudar a executar as atividade e usar o aplicativo, as vantagens e dificuldades no uso do aplicativo, a utilidade dos alertas como lembretes para a realização das atividades em casa, a motivação para continuidade no uso do aplicativo, sugestões de modificações e melhorias do mesmo, uma avaliação sobre o treinamento oferecido para o uso inicial do aplicativo e a pertinência das atividades solicitadas; 2) um questionário com 28 afirmações sobre as quais o participante deveria fazer uma avaliação acerca da sua satisfação ao interagir com o aplicativo e sobre aspectos de usabilidade do mesmo (e.g. "Eu achei fácil inserir dados no aplicativo", "Eu usaria este aplicativo com frequência"). O questionário foi elaborado com base no SUS (System Usability Scale) (BROOKE, 1996) e no QUIS (Questionnaire for User Interaction Satisfaction) (CHIN et al., 1988), instrumentos utilizados na literatura da área de Interação Humano-Computador (IHC) para avaliação de usabilidade e satisfação no uso, respectivamente; e 3) um Questionário de Experiência de Uso (tradução em português do User Experience Questionnaire -UEQ) (LAUGWITZ; HELD; SCHREPP, 2008). Esse questionário teve como objetivo avaliar a experiência dos participantes na interação com o aplicativo em termos de sentimentos, considerando diferentes aspectos do mesmo e cinco fatores: Atratividade, Perspicácia, Eficiência, Confiabilidade, Estimulação e Inovação.

\section{3 Atividades programadas}

Foram programadas sete atividades para serem realizadas ao longo de duas semanas consecutivas: três na primeira semana e quatro na segunda. Os participantes receberam alertas sonoros diários para a execução das atividades, assim algumas foram solicitadas mais de uma vez, no entanto, uma mesma atividade nunca era solicitava em dias consecutivos. Os conteúdos foram previamente aprendidos durante as aulas dos cursos de alfabetização e letramento digital.

A Tabela 3 apresenta a descrição das atividades programadas, a frequência de solicitação de execução e os horários do disparo de alertas para a execução das mesmas. Os horários programados para os alertas foram definidos pelos participantes com base em seu tempo livre para realizar as atividades. Os mesmos elegeram o início das manhãs e início das noites como os momentos mais adequados. 
Em cada atividade os participantes deveriam executar de acordo com as instruções apresentadas. As atividades eram idênticas aos usuários de smartphones e tablets sempre que possível. Quando isso não era possível, as atividades eram projetadas para serem equivalentes em termos de função (como nas atividades A1 e A5 apresentadas na Tabela 3). A verificação da execução era feita automaticamente (A) por meio de dados enviados pelo sistema ou manualmente $(\mathrm{M})$, por meio de checagem presencial pelos professores do curso nas aulas subsequentes ao estudo.

Tabela 3 - Detalhamento das atividades programadas

\begin{tabular}{|c|c|c|c|c|c|}
\hline \multirow{2}{*}{ Semana } & \multicolumn{2}{|c|}{ Atividade } & \multirow{2}{*}{$\begin{array}{l}\text { Frequência/ } \\
\text { dia da semana }\end{array}$} & \multirow{2}{*}{ Horário } & \multirow{2}{*}{ Verificação } \\
\hline & $\mathrm{N}$ & Conteúdo & & & \\
\hline \multirow[t]{4}{*}{1} & $\mathrm{~A} 1$ & $\begin{array}{l}\text { Fazer uma Ligação: } \\
\text { - Tablet: usando Skype. } \\
\text { - Smartphone: usando a } \\
\text { lista de contatos. }\end{array}$ & $\begin{array}{l}2 \text { vezes } \\
\text { (Seg; Qui) }\end{array}$ & 19:00 & M \\
\hline & $\mathrm{A} 2 \mathrm{a}$ & $\begin{array}{l}\text { - Baixar aplicativo da Play } \\
\text { Store }\end{array}$ & 1 vez (Qua) & $19: 30$ & M \\
\hline & $\mathrm{A} 2 \mathrm{~b}$ & $\begin{array}{l}\text { - Interagir com o aplicativo } \\
\text { baixado }\end{array}$ & $\begin{array}{l}2 \text { vezes } \\
\text { (Sex; Sab) }\end{array}$ & & \\
\hline & $A 3$ & $\begin{array}{l}\text { Enviar uma foto de si } \\
\text { mesmo (selfie) pelo sistema } \\
\text { ESPIM }\end{array}$ & $\begin{array}{l}2 \text { vezes } \\
\text { (Ter; Dom) }\end{array}$ & $8: 30$ & A \\
\hline \multirow[t]{4}{*}{2} & A4 & $\begin{array}{l}\text { Enviar um vídeo deles } \\
\text { mesmos desejando um } \\
\text { bom dia pelo sistema } \\
\text { ESPIM. }\end{array}$ & $\begin{array}{l}2 \text { vezes } \\
\text { (Seg; Qui) }\end{array}$ & $8: 00$ & A \\
\hline & A5 & $\begin{array}{l}\text { Compartilhar uma foto } \\
\text { - Tablet: no grupo da sala } \\
\text { do Facebook } \\
\text { - Smartphone: no grupo da } \\
\text { sala do Whatsapp }\end{array}$ & $\begin{array}{l}3 \text { vezes } \\
\text { (Ter; Sex; Sab) }\end{array}$ & 19:00 & M \\
\hline & A6 & $\begin{array}{l}\text { A6 - Selecionar em meio a } \\
\text { uma lista os assuntos vistos } \\
\text { no curso e escrever quais } \\
\text { assuntos foram mais fáceis }\end{array}$ & 1 vez (Qua) & $19: 30$ & A \\
\hline & A7 & $\begin{array}{l}\text { Escrever se precisou de } \\
\text { ajuda para usar o aplica- } \\
\text { tivo, dificuldades e pontos } \\
\text { positivos }\end{array}$ & 1 vez (Dom) & $8: 30$ & A \\
\hline
\end{tabular}

Fonte: Elaborada pelos autores. 
As atividades A1, A2, A3, A4 e A5 foram projetadas para que os participantes praticassem conteúdos aprendidos em sala de aula em casa. A6 foi projetada para coletar dados sobre as percepções dos participantes sobre o conteúdo aprendido e A7, para entender como os participantes avaliaram o aplicativo do ESPIM em termos de aspectos positivos e negativos.

Mais especificamente, A1 buscava estimular a comunicação, visando as habilidades necessárias para realizar chamadas de voz. Para o grupo de smartphones, os participantes foram solicitados a fazer uma ligação telefônica para o professor do curso. Para isso, eles precisavam encontrar o número do professor em sua lista de contatos e fazer a ligação. Para o grupo de tablets, os participantes deveriam fazer uma chamada usando Skype para o professor, exigindo que eles localizassem o aplicativo Skype em seus dispositivos, encontrassem o contato do professor e fizessem a chamada. Essa atividade foi solicitada duas vezes na primeira semana do estudo. A execução da atividade foi verificada manualmente pelos professores, que registraram as chamadas recebidas dos alunos.

A A2 foi destinada para o treino de habilidades envolvidas para: a) baixar novos aplicativos (como entrar na Play Store, encontrar um aplicativo específico e instalá-lo), e b) usar o aplicativo recém-instalado, localizando o aplicativo no dispositivo e interagindo com ele. $\mathrm{O}$ aplicativo a ser baixado era o "jogo do tomate", desenvolvido para ensinar usuários idosos a realizar ações de seleção por meio do toque e arrastar. Essa atividade foi solicitada três vezes na primeira semana do estudo, para que os participantes tivessem mais do que uma oportunidade para realizar a atividade. Como os participantes não poderiam baixar o mesmo aplicativo mais de uma vez, a partir da segunda solicitação dessa atividade, a primeira pergunta foi de múltipla escolha: "Você já baixou o jogo do tomate?", e os participantes deveriam selecionar "Não" ou "Sim" como resposta. No caso de uma resposta negativa os participantes eram instruídos a primeiro baixar o jogo (A2a) e, em seguida, jogar (A2b). No caso de resposta afirmativa, os participantes eram instruídos a jogar o jogo (A2b). Tal atividade demonstra a funcionalidade de fluxos de interação diferentes no sistema ESPIM, de acordo com a resposta fornecida pelos participantes.

As atividades A3 e A4 visavam treinar os participantes sobre o uso da câmera frontal para tirar fotos e gravar vídeos. As habilidades envolvidas eram, localizar o aplicativo da câmera, escolher entre a função de foto ou vídeo, alternar da câmera traseira para a câmera frontal e tirar uma selfie ou gravar um vídeo. A3 foi solicitada duas vezes na primeira semana e A4, duas vezes na segunda semana. A execução dessas atividades foi verificada automaticamente no sistema pelos dados enviados por meio do aplicativo. 
A atividade A5 também tinha como objetivo observar aspectos de comunicação, assim como A1, no entanto, por meio de aplicativos de rede social. Para o grupo de smartphones, os participantes foram solicitados a compartilhar uma foto de sua galeria no grupo do WhatsApp do curso. As habilidades necessárias eram: encontrar o aplicativo WhatsApp em seus dispositivos, encontrar o grupo do curso, selecionar a função "anexar", selecionar "galeria", escolher uma foto da galeria e enviar a foto. Para os grupos de tablets, as habilidades necessárias eram: encontrar o aplicativo do Facebook em seus dispositivos, encontrar a página do curso no Facebook, selecionar a função "postar foto", selecionar uma foto da galeria e publicá-la. Essas atividades foram solicitadas três vezes na segunda semana do estudo.

A6 teve como objetivo verificar se os participantes lembravam quais conteúdos foram aprendidos durante o curso apresentando assim, uma lista para escolha. Nem todos os conteúdos listados faziam parte do curso. Esse tipo de pergunta permitia verificar se os participantes entenderam o conceito de selecionar mais de uma opção e também verificar se os mesmos estavam atentos ao conteúdo ministrado em sala. Na sequência, os participantes foram solicitados a digitar um pequeno texto escrito sobre quais foram os conteúdos mais facilmente aprendidos. A execução da atividade foi verificada automaticamente pelo sistema.

Finalmente, A7 foi uma pergunta aberta aos participantes sobre suas impressões acerca do uso do aplicativo. Os participantes deveriam digitar um texto para respondê-la. Esta atividade foi solicitada apenas uma vez e no último dia de coleta de dados do estudo e avaliava aspectos como destreza no uso do teclado virtual.

A Figura 3 ilustra as telas do aplicativo do ESPIM para o conjunto de intervenções referentes à tarefa A2 supracitada, e a maneira como cada fluxo era visualizado pelos participantes em seus dispositivos. A intervenção considera dois fluxos de ações possíveis: O Fluxo 1 é representado na parte superior, e o Fluxo 2, na parte inferior. A intervenção programada tinha o objetivo de treinar a tarefa de baixar aplicativos e usar o aplicativo baixado. A primeira interação apresentava ao participante uma questão de múltipla escolha "Você baixou o jogo do tomate?", com duas possibilidades de resposta ("Sim" e "Não") e tal resposta definia o fluxo de ações seguintes. Caso a resposta fosse afirmativa ("Sim"), o participante era direcionado para uma mensagem de texto que o incentiva a procurar o jogo em seu dispositivo e jogá-lo (Fluxo 1). Em seguida, a intervenção era finalizada. Caso o participante respondesse negativamente ("Não"), ele recebia uma instrução textual contendo um link (disponibilizado no botão "Iniciar" - ver Figura 3) que o direcionava para o aplicativo na loja 
virtual Play Store, no qual ele deveria usar o campo de busca com a string "Jogo do Tomate" para encontrar o jogo, baixá-lo e, então, jogá-lo (Fluxo 2). Depois desses passos, o programa interventivo era encerrado.

Figura 3 - Visualização das telas de intervenção no dispositivo móvel do participante
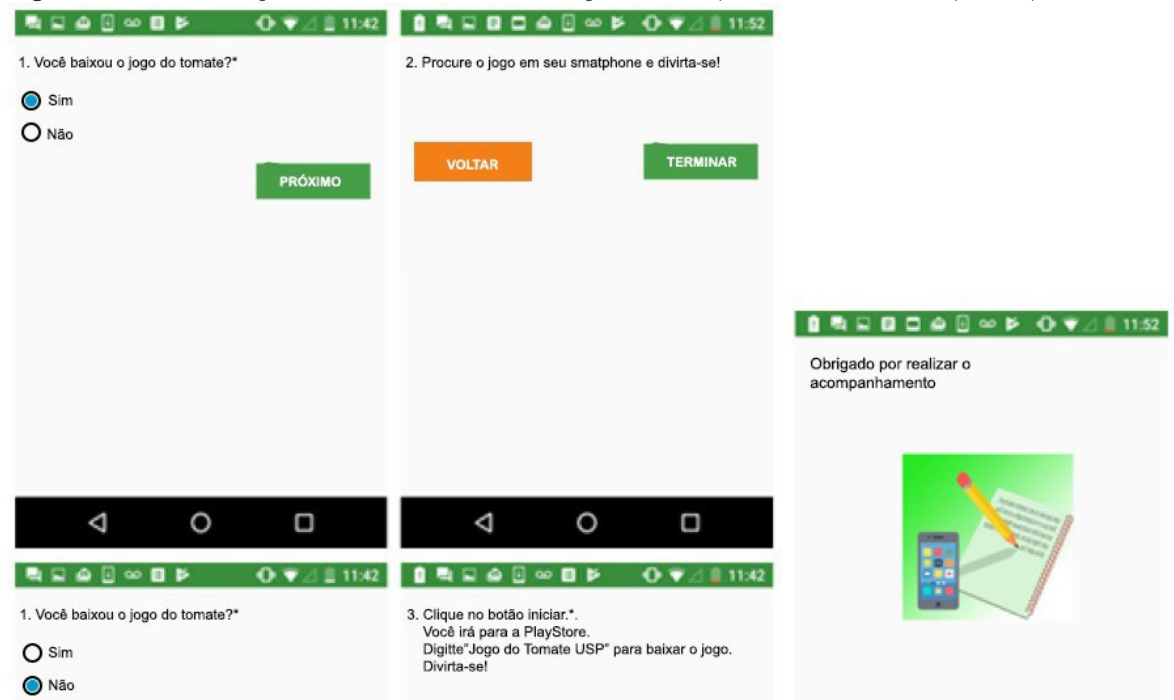

PRÓXIMO

INICIAR

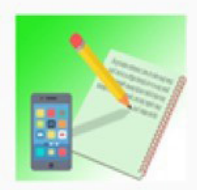

O Năo

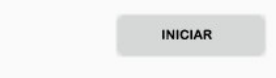

4

O

口

\begin{tabular}{|l|l} 
VOLTAR & TERMINAR \\
\hline
\end{tabular}

\section{$\triangleleft \quad 0 \quad \square$}

\section{4}

口

Fonte: Elaborada pelos autores.

Nota: A parte superior da figura apresenta o Fluxo 1 de atividades e a inferior, o Fluxo 2. Os dois fluxos são encerrados em uma tela comum que sinaliza o final da atividade, demonstrado pela parte à direita. 


\subsection{Procedimentos}

A coleta de dados por meio do aplicativo foi programada para ocorrer remotamente, usando o dispositivo do participante, com alarmes disparando em períodos em que os idosos provavelmente estivessem em suas residências (início da manhã e da noite). As aulas dos cursos de alfabetização e letramento digital foram realizadas nas respectivas instituições. Nessas aulas ocorriam a verificação da realização de algumas das atividades programadas, validando assim, a interação ou não com as mesmas.

O aplicativo do sistema ESPIM foi instalado nos dispositivos de cada aluno e de cada professor/monitor dos cursos de extensão. Após instalado, os idosos receberam um treinamento individual de uso do aplicativo. No treinamento, os idosos aprenderam a usar os botões que guiavam o fluxo das telas das atividades, por exemplo: "Próximo" e "Voltar" (vide Figuras 2 e 3), que permitiam avançar ou retornar às telas anteriores; "Gravar Vídeo" e "Tirar Foto", que permitiam o uso da câmera para filmagem e fotografias; e "Terminar", que encerrava uma interação em andamento. Os idosos também foram treinados para realizar ações que seriam requisitadas nas atividades, tais como: selecionar por meio de toque uma opção de texto (resposta a uma lista); produzir pequenos textos (resposta a questões abertas).

As instruções fornecidas aos idosos apresentavam o aplicativo como um "ajudante digital" que poderia estimular a interação com os dispositivos para além da sala de aula. Os participantes foram informados de que receberiam uma mensagem de alerta por dia, por meio do aplicativo, para que eles pudessem realizar alguma atividade relacionada aos conteúdos já aprendidos durante o curso. Os alertas e atividades de reforço seriam enviados por um período de duas semanas consecutivas (15 dias).

Os participantes podiam executar as atividades de duas maneiras: 1) esperar pelo alerta do aplicativo; ou 2) autoiniciar as atividades acessando o aplicativo em seus dispositivos em momento oportuno. Também foram instruídos de que a verificação da execução das atividades seria feita automaticamente pelo sistema ou por meio da verificação dos monitores/professores em sala de aula, na semana seguinte do uso do aplicativo. Após o término da coleta de dados por meio do aplicativo ESPIM, foram agendados encontros individuais com os idosos com o objetivo de obter dados subjetivos acerca da experiência de uso e da interação com o aplicativo. 
Os resultados são apresentados considerando a execução das atividades em ambiente natural pelo idoso (usando o sistema ESPIM), e os dados coletados por meio dos questionários e entrevistas utilizados para avaliar o requisito de usabilidade e a experiência de uso dos participantes ao interagir com o aplicativo móvel.

\subsection{Sobre a execução das atividades}

A Figura 4 apresenta o número individual de vezes em que cada participante executou com sucesso cada atividade solicitada durante o estudo.

Figura 4 - Número de execuções de cada atividade por participante

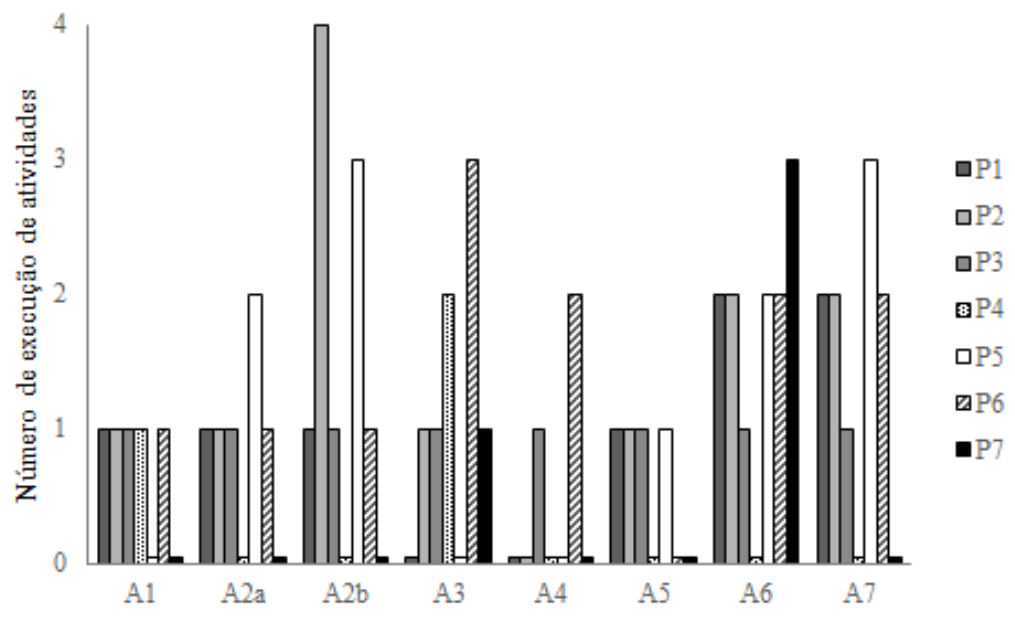

Fonte: Elaborada pelos autores.

Os dados mostram que, em geral, os participantes se engajaram na execução das atividades na maioria das vezes. Participante P3 realizou $100 \%$ das atividades pelo menos uma vez, e P2 e P6 realizaram 87,5\% das atividades, P1 e P5 realizaram 62,5\%. Apenas P4 e P7 apresentaram menor taxa de engajamento com as atividades (25\%). No caso de P4, sua participação no estudo foi interrompida na segunda semana, devido a uma queda, seguida de hospitalização na semana 2. Como os participantes poderiam auto-iniciar as atividades, 
pode-se observar que alguns participantes interagiram algumas atividades mais vezes do que o programado e solicitado pelo aplicativo.

As atividades realizadas um maior número de vezes foram: A2 (baixar um aplicativo e interagir com ele), A3 (tirar uma selfie), A5 (compartilhar uma foto usando o WhatsApp ou Facebook). Essas atividades envolviam a produção de mídia e a interação com recursos e aplicativos externos, o que podem ter sido mais atraentes para os participantes. A atividade com o menor engajamento foi A4, que exigiu a gravação de um vídeo, habilidade que demandava mais habilidade e consolidação do conteúdo ensinado. O número total de interações dos participantes ao longo da primeira e da segunda semanas do teste é apresentado na Figura 5. Os resultados são apresentados separadamente para cada instituição porque a ordem em que as atividades foram solicitadas nem sempre foi a mesma entre as instituições, devido aos diferentes horários de aulas por semana.

Figura 5 - Número total de interações dos participantes durante as duas semanas do estudo

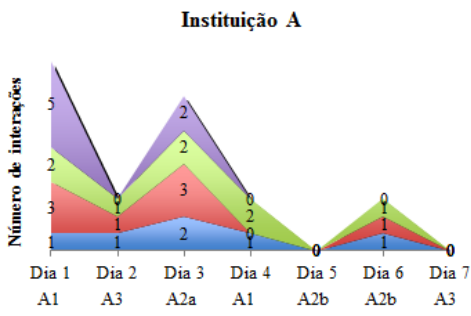

Semana 1

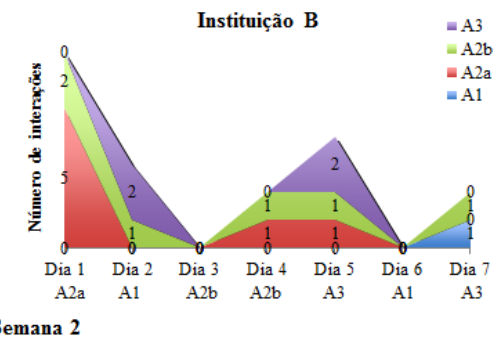

Instituição A

Instituição B

$=\mathrm{A} 7$

$=\mathrm{A} 6$
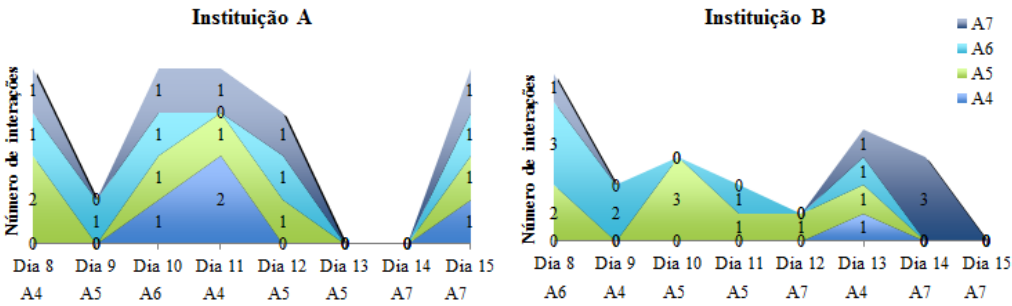

Fonte: Elaborada pelos autores.

Nota: $O$ eixo horizontal representa o dia de coleta e a atividade que foi programada para ser solicitada para os participantes pelo aplicativo.

Pode-se observar que houve um grande número de execução das atividades logo no primeiro dia, o que poderia indicar um efeito de novidade, ou seja, os participantes se interessaram em explorar as atividades pelo aplicativo 
uma vez que essa era uma nova prática. Mesmo assim, os participantes continuaram a interagir com as atividades durante as semanas que seguiram, por pelo menos cinco dias. Além disso, era comum os participantes interagirem com mais de uma atividade por dia. Também foram observados dois padrões de interação com as atividades: 1) responder à atividade solicitada em um dia específico; ou 2) selecionando e autoiniciando uma das atividades disponíveis. O primeiro padrão é representado pelos dados no Dia 1 da Instituição B. Nesse caso, a atividade solicitada foi A2a (baixar aplicativo da Play Store) e a maioria das interações dos participantes com o sistema envolveu essa atividade. Um padrão semelhante também é observado nos dias 5 e 10 e 14, na Instituição B e no Dia 3, na Instituição A. O segundo padrão de interação, no entanto, foi mais comum e representou a maioria das interações dos participantes. No Dia 1, Instituição A, os participantes interagiram com todas as atividades disponíveis, apesar de apenas a atividade $\mathrm{A} 1$ ter sido solicitada. $\mathrm{O}$ mesmo padrão pode ser observado também no Dia 10, Instituição A e no Dia 13, Instituição B. Esse padrão de interação indica que os participantes agiram de forma autônoma usando o aplicativo, que era totalmente novo para eles, e escolhendo quais atividades executariam e quando as fariam. Esse comportamento pode indicar que o aplicativo era simples o suficiente para estimular os participantes a explorá-lo em vez de esperar passivamente que o aplicativo direcionasse a interação.

\subsection{Sobre a interação com o aplicativo}

\subsubsection{Entrevista semiestruturada}

Os dados coletados por meio da entrevista semiestruturada acerca da experiência de uso do aplicativo foram sumarizados nas seguintes categorias: 1) frequência de engajamento na interação com o dispositivo fora da sala de aula; 2) aspectos positivos do aplicativo; 3) dificuldades enfrentadas no uso do aplicativo e sugestões de melhorias; 4) envolvimento de outras pessoas para o uso do aplicativo; e 5) motivação para continuidade de uso do aplicativo.

Com relação à primeira categoria, a frequência de engajamento dos participantes com os dispositivos móveis foi acessada por meio de autorrelato. Seis participantes relataram aumento da frequência no uso do tablet ou smartphone após a introdução do aplicativo: cinco deles relataram aumento de uso de três a quatro vezes na semana e um participante relatou aumento de uma a duas vezes na semana. Somente um participante (P3) relatou que a frequência de 
uso do smartphone permaneceu inalterada. É interessante ressaltar que tal participante apresentava, dentre todos, maior frequência de uso do dispositivo e maior variabilidade no uso de funções do mesmo antes do início do estudo.

Dentre os aspectos positivos associados ao uso do aplicativo, todos os participantes fizeram comentários acerca do aumento de oportunidades de treino dos conteúdos aprendidos em sala. Seguem alguns trechos literais de falas dos participantes que ilustram tal aspecto: P1 - "Foi importante para ratificar o que realmente aprendemos e somos capazes de usar"; P5 - "Serviu como incentivo para treinar em casa". Além disso, os participantes mencionaram que o treino em casa evita que o conteúdo aprendido seja esquecido e estimula que eles tentem resolver os problemas e dificuldades de uso de maneira mais independente (P2 - "Me forcei um pouco a aprender"; P3 - "Faz despertar o cérebro. Despertar o pensar").

Um segundo aspecto positivo mencionado foi o recebimento de alertas sonoros como lembretes para a realização das atividades. O P5 relatou que "[os alertas] foram muito úteis, pois sabia que depois que tocava tinha que fazer uma atividade. Ajudou especialmente no final de semana, em que a rotina é diferente".

Além da execução das atividades subsequente aos alertas enviados, todos os participantes relataram ter, em algum momento, autoiniciado atividades. A possibilidade de realizar as atividades em momentos mais convenientes também foi apontada como um aspecto positivo. Por exemplo, os participantes P1, P2 e P4 preferiam executar as tarefas no período da noite, ainda que o alerta sonoro estivesse programado para ser acionado na parte da manhã. Nesses casos, quando o alerta tocava pela manhã eles o ignoravam e interagiam com o aplicativo mais tarde, quando lhes era mais conveniente.

Outro aspecto levantado por quatro dos participantes foi que o uso do aplicativo possibilitou oportunidades de auto-avaliação de desempenho e do grau de autonomia que apresentavam com relação às atividades solicitadas. A participante P5 relatou: "Gostei de usar e saber que eu estou conseguindo fazer. Me senti bem!". Os participantes também mencionaram que o uso do aplicativo se tratava de um compromisso que seria verificado: P1 — "Ter tarefa pra fazer em casa fora da aula é importante para reforçar [...]. Antes a monitora pedia, mas eu não fazia. Passei a fazer depois do uso do aplicativo porque tinha onde registrar e tinha cobrança".

Apesar da maior parte dos relatos ter sido positiva, alguns participantes apresentaram dificuldades na interação com o aplicativo e fizeram sugestões de modificações. Com relação ao design, P1 apontou que os botões e o texto não se destacavam tanto do fundo e P4 sugeriu textos e botões maiores, para 
facilitar o toque e a digitação. Sobre o registro de mídias (vídeos e fotografias), P6 sugeriu que houvesse uma opção para visualizar o vídeo/fotografia antes de realizar o envio.

Outras dificuldades relatadas não foram relacionadas ao uso do aplicativo, mas sim ao manuseio dos dispositivos. Por exemplo, os participantes P2 e P7 tiveram dificuldades com o uso da câmera de seus dispositivos, em especial, em alternar entre as câmeras frontal e traseira. Essa dificuldade indicou necessidade de realizar mais treino em sala de aula para promover aprendizagem duradoura sobre o uso correto das duas câmeras disponíveis no dispositivo.

Em relação a recorrer a outras pessoas para ajudar a usar o aplicativo, três dos sete participantes recorreram a alguém somente uma vez diante de alguma dificuldade. P2 recorreu à filha para ajudá-la na primeira solicitação da atividade A3, que necessitava de câmera fotográfica: "Pedi ajuda uma vez, depois não quis mais. Me forcei um pouco a aprender sozinha". P6 recorreu à neta na primeira solicitação da atividade A4 de gravação de vídeo e P4 recorreu ao filho no momento em que seu dispositivo apresentou mensagem de erro por falta de espaço na memória (A3).

Em termos de adesão ao uso do aplicativo, todos os participantes relataram que continuariam usando o aplicativo como seus "ajudantes digitais" e que recomendariam o uso aos colegas de sala também. Sobre a quantidade de atividades solicitadas, uma vez que idosos em geral apresentam menor familiaridade e experiência com a tecnologia quando comparados com outros grupos etários (HAUK; HÜFFMEIER; KRUMM, 2018), neste estudo houve uma preocupação de que a demanda das tarefas não fosse excessiva. Assim, a solicitação para fazer atividades foi limitada a uma por dia. Seis dos sete participantes relataram que gostariam de ter mais atividades para cumprir em casa durante a semana e, de que gostariam de ter usado o aplicativo como apoio ao uso dos dispositivos fora da sala de aula desde o início do curso. P3 relatou: "Achei que foi tímida a interferência do aplicativo [...]. Foi muito curto. Queria que tivesse mais atividades"; P4: “Eu queria ter usado isso desde o início do curso [...] e queria usar mais".

Os participantes julgaram as instruções das atividades a serem executadas sucintas, claras e de fácil compreensão. A maior parte relatou sentimentos de autoconfiança ao realizar as atividades solicitadas. Eles também valorizaram interagir com um aplicativo com fins educativos. P2 comentou positivamente sobre o aspecto de personalização do aplicativo, uma vez que as atividades solicitadas foram especialmente programadas para estimular o uso e monitorar a aprendizagem daquele grupo de alunos em específico. 
Os itens do questionário de usabilidade foram agrupados em três grandes categorias: 1) facilidade de uso, 2) autonomia, e 3) sentimentos gerados durante o uso. Os resultados são apresentados na Figura 6. Os resultados podiam variar entre -2 e 2 pontos. Observa-se que a pontuação média para cada aspecto avaliado dentro das três categorias foi positiva em todos os casos. A média geral dos itens que avaliavam facilidade de uso foi de 1,03 (MD =2,0), indicando que os participantes consideraram fácil usar e aprender a usar o aplicativo. A maior pontuação foi a de "facilidade para navegar entre menus e telas do aplicativo", com escore máximo de 2,0 pontos. Esse resultado reflete os esforços feitos na fase de concepção e design do aplicativo, de forma que o mesmo fosse o mais intuitivo possível, para minimizar erros ou execução de passos desnecessários na interação. Assim, a navegação entre telas apresentava um número mínimo de botões e possibilidades de interação, com fluxos claros dos passos para seguir adiante em próximas telas ou retornar às telas anteriores (vide Figura 3).

Figura 6 - Pontuação média da avaliação dos participantes idosos do aplicativo em termos de facilidade de uso (A), autonomia (B) e sentimentos gerados (C)

A) Facilidade de Uso

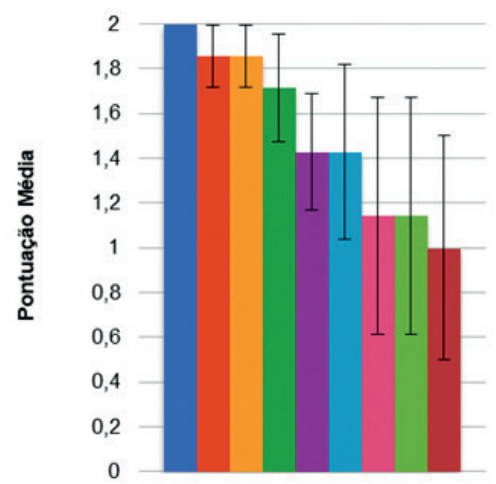

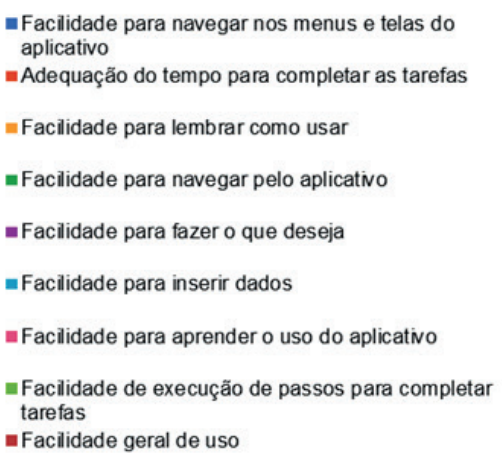




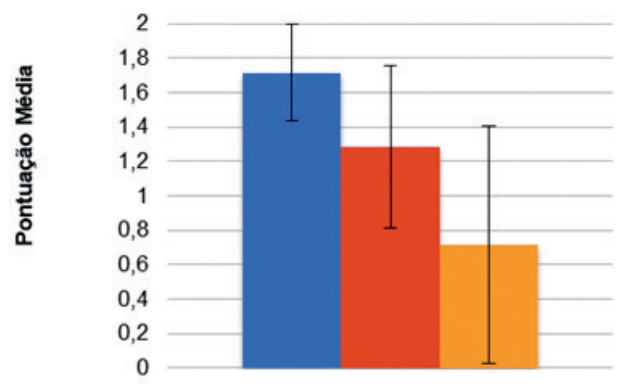

- Competência e controle no uso

n Comportamento previsivel do aplicativo

Independência no uso

C) Sentimentos Gerados

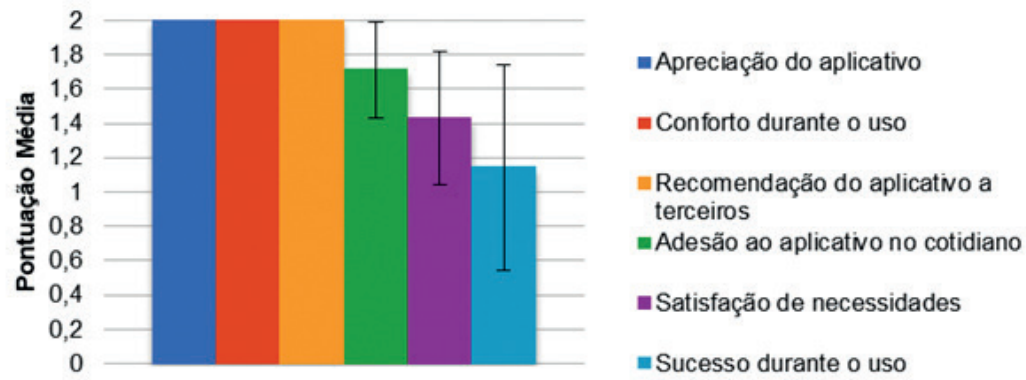

Fonte: Elaborada pelos autores.

Em termos de autonomia, a média geral dos itens que avaliavam tal fator foi de 1,23 pontos ( $\mathrm{MD}=2,0)$. Os participantes se consideraram competentes e no controle durante o uso do aplicativo. Os sentimentos gerados durante a interação com o aplicativo foram bastante positivos, com média de 1,71 pontos $(\mathrm{MD}=2)$. Houve pontuação máxima para as afirmações "Eu gostei de usar este aplicativo", "Eu me senti confortável usando este aplicativo" e "Eu recomendaria o uso deste aplicativo a outras pessoas", refletindo os dados levantados na entrevista semiestruturada.

\subsubsection{Questionário Experiência de Uso (UEQ)}

A Figura 7 apresenta as pontuações medianas dos participantes para cada par de adjetivos/conceitos bipolares avaliadas pelo UEQ. Os valores poderiam variar entre - 3 e 3 pontos. Observa-se que a avaliação dos participantes 
se aproximou majoritariamente em torno do polo positivo, entre 2 e 3 pontos. Os fatores mais bem avaliados foram confiabilidade $(\mathrm{M}=1,9 ; \mathrm{MD}=2,5)$, eficiência $(\mathrm{M}=2 ; \mathrm{MD}=2)$, perspicácia $(\mathrm{M}=1,9 ; \mathrm{MD}=2)$ atratividade $(\mathrm{M}=1,8 ; \mathrm{MD}=2)$ e inovação $(\mathrm{M}=1,7 ; \mathrm{MD}=2)$, indicando que os participantes consideraram o aplicativo fácil de entender, compreensível, amigável, interessante, confiável, eficiente e inovador. Os participantes também julgaram o aplicativo inovador e estimulante $(\mathrm{M}=1,7 ; \mathrm{MD}=2)$.

Figura 7 - Valores medianos para cada par de adjetivos/conceitos bipolares para os fatores atratividade, perspicácia, inovação, estimulação, confiabilidade e eficiência avaliados pelo UEQ

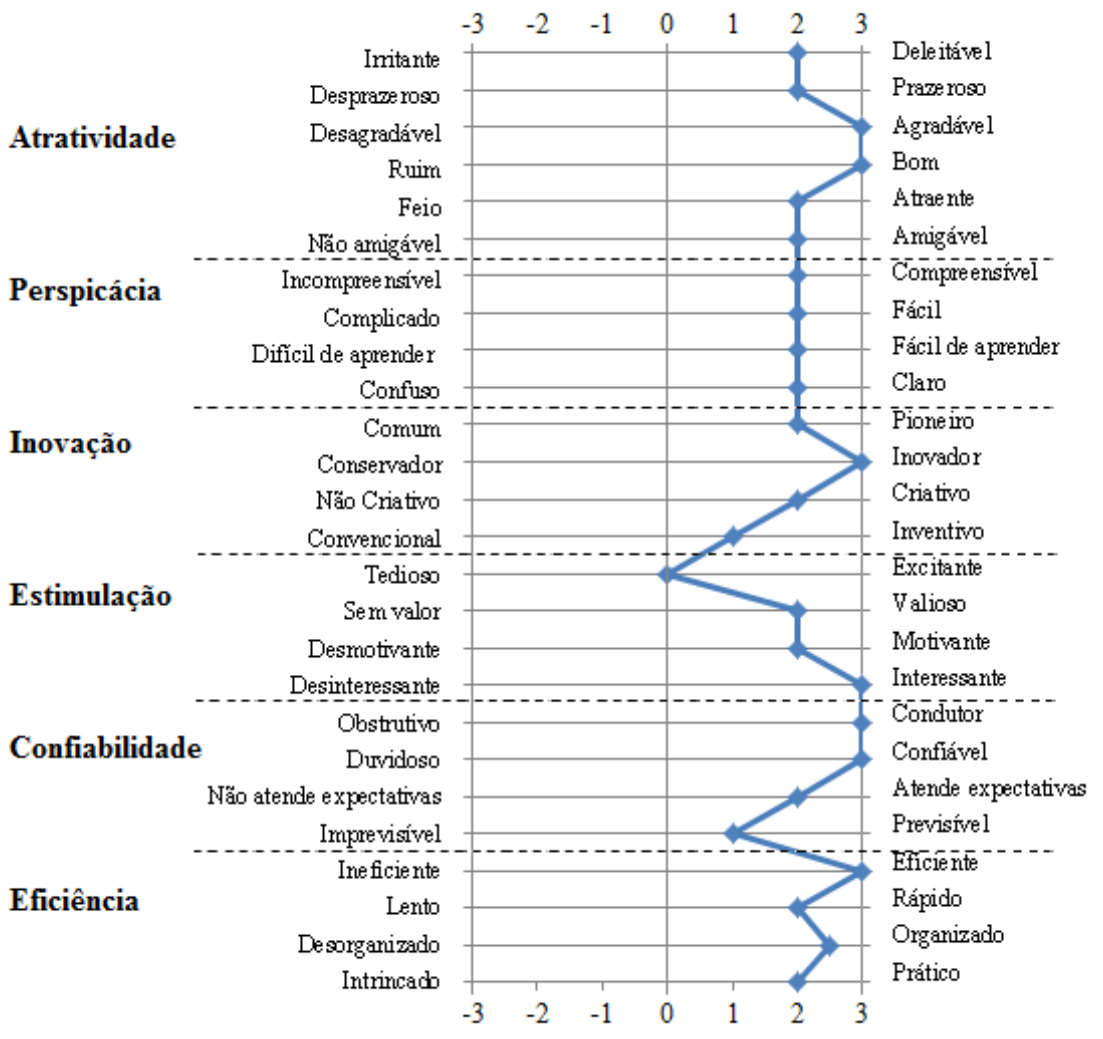

Fonte: Elaborada pelos autores. 
Uma das grandes dificuldades para o desenvolvimento de atividades de alfabetização e letramento digital direcionado às pessoas idosas é garantir a prática e a generalização de repertórios aprendidos em sala de aula ao cotidiano dos alunos. Os resultados obtidos com este estudo foram encorajadores quanto à inclusão do sistema ESPIM como recurso para o estímulo ao engajamento e para o monitoramento do uso de dispositivos móveis entre pessoas idosas participantes de programas de alfabetização e letramento digital. Os participantes prontamente adotaram o uso do sistema como forma de auxílio para a execução de atividades em casa relacionadas aos conteúdos aprendidos em sala de aula. Dentre as atividades com maior engajamento, destacaram-se as que favoreciam o envolvimento com as mídias sociais, dado corroborado por Hauk, Hüffmeier e Krumm (2018). Esses autores verificaram que dentre as atividades mais realizadas por idosos, no que diz respeito à adesão a tecnologias, estão aquelas que visam comunicação, a busca por apoio social, lazer e entretenimento.

A experiência no uso de aplicativo foi positiva para todos os participantes. Eles consideraram o aplicativo fácil de aprender a usar mesmo sem assistência de outra pessoa, associaram sentimentos positivos ao uso do mesmo e não se sentiram incomodados pelas interrupções diárias feitas pelo aplicativo. Além disso, recomendariam aos amigos e gostariam de ter tido mais oportunidades de realizar as "tarefa-de-casa" usando o aplicativo. Esses são bons indicadores de que o uso do aplicativo do ESPIM seria bem recebido e adotado pela população idosa, em geral, com o objetivo de promover a alfabetização e letramento digital em uma escala maior e período de tempo maiores.

De acordo com os relatos dos participantes, o uso do aplicativo aumentou o engajamento com os dispositivos móveis em casa, de maneira geral, e também para treinar os conteúdos e habilidades específicos aprendidos em sala de aula. Intervenções que usam tecnologia para testar a eficácia e a aceitação de plataformas e aplicações tecnológicas por pessoas idosas são relatadas por Kuerbis et al. (2017), que demonstraram a viabilidade e eficácia de plataformas que intervêm em comportamentos de saúde por meio de tecnologias móveis (RUIZ et al., 2011; DEAR et al., 2013). Os resultados deste estudo corroboram com tais relatos e os estendem à adoção de soluções tecnológicas voltadas para fins educacionais por idosos.

Uma vez que o segmento idoso representa uma proporção menor de usuários de tecnologias, muitas vezes são criados estereótipos, sendo descritos como tecnofóbicos (TSAI; SHILLAIR; COTTEN, 2017). Há dados, no entanto, 
apoiando que essa população também pode adotar e abraçar a tecnologia, sendo que há um número significativo de adultos mais velhos que usam tecnologias para buscar informações e que apresentam atitudes positivas em relação à tecnologia móvel (KUERBIS et al., 2017; PARK; MCLAUGHLIN, 2010). Os resultados aqui apresentados corroboram com tal tendência e com a atitude positiva dos idosos em relação à adoção de tecnologia: nossos participantes mostraram alta adesão ao uso de uma tecnologia de solução de software até então desconhecida por eles (aplicativo ESPIM) como ferramenta de suporte para o uso de dispositivos móveis no cotidiano. O uso do aplicativo motivou uma maior participação na realização de atividades sobre conteúdo e sobre as práticas aprendidas em sala de aula sobre o uso de smartphones e tablets. De acordo com o relato dos participantes o uso do aplicativo foi mais eficaz do que incentivos e instruções verbais para que eles praticassem fora da sala de aula. Além disso, nossos participantes foram resilientes ao enfrentar dificuldades e frustrações no uso dos dispositivos e do aplicativo.

Dentre os desafios às práticas de letramento digital, destaca-se, segundo Kuerbis et al. (2017), a pouca autoconfiança de idosos sobre aprender algo novo e a pouca associação a repertórios educativos anteriores. Para Zandbergen (2015), os principais problemas apontados por idosos que utilizam os dispositivos móveis estão relacionados com a falta de conhecimento tecnológico no manuseio dos aplicativos, o que influencia diretamente na falta de persistência desse público no uso dos dispositivos móveis. Tal resistência no uso da tecnologia na velhice pode ser explicada pela Teoria da Seletividade Socioemocional (LOCKENHOFF; CARSTENSEN, 2004). Dentre os principais pontos, essa teoria discute que com a percepção do processo de envelhecimento e de que há menos tempo disponível, as pessoas passam a priorizar metas orientadas para o presente e de maior conteúdo emocional, como relações sociais íntimas, família e atividades emocionalmente satisfatórias, sobre aquelas metas voltadas para o futuro, como a aquisição de informações e melhora de desempenho. A maior parte do desenvolvimento de tecnologias, no entanto, objetiva justamente as prioridades orientadas para o futuro, melhorias de desempenho, efetividade e eficácia (LEE; KAO; YANG, 2014). Assim, a maioria das tecnologias disponíveis pode não atender aos objetivos orientados para o presente dos idosos, levando a uma menor adesão a elas.

No entanto, estudos mostram que essas barreiras podem ser superadas se os idosos perceberem relevância no uso de um recurso tecnológico (HAUK; HÜFFMEIER; KRUMM, 2018). Em nosso estudo, uma vez que os idosos perceberam o uso do aplicativo como relacionados a objetivos do presente, não houve dificuldade de adesão dos participantes; pelo contrário, eles prontamente 
aceitaram e adotaram a tecnologia. De acordo com o modelo denominado Technology Acceptance Model - TAM (DAVIS, 1989) as pessoas aceitam e adotam uma nova tecnologia baseada em dois fatores principais: facilidade de uso e utilidade percebida (CHEN; CHEN; YEN, 2011). Este estudo de viabilidade mostrou que o aplicativo ESPIM foi caracterizado pelo público-alvo como simples e intuitivo, permitindo que os mesmos o usasse de forma autônoma. Além disso, eles perceberam o uso do aplicativo como valioso para suas atividades no curso de alfabetização e letramento digital e, para o uso de tecnologias em geral, o que pode fortalecer sua motivação para usar o novo aplicativo de forma mais prolongada.

\section{Considerações finais}

O uso de um recurso tecnológico aliado às práticas pedagógicas, assim como o planejamento do conteúdo de cada atividade, e a programação das condições de ensino, continuam sendo importantes para aumentar a efetividade da aprendizagem tecnológica pelos idosos (JONES-KAVALIER; FLANNIGAN, 2008). O uso do aplicativo do sistema ESPIM desempenhou um papel importante visando à generalização do conteúdo aprendido em sala de aula ao ambiente natural e cotidiano, de modo que essas tecnologias podem ser de fato utilizadas por essa população, proporcionando, assim, a manutenção da aprendizagem. A verificação, manutenção e estimulação de repertórios aprendidos no ambiente natural é um desafio para pesquisadores com interesses aplicados, no entanto, é possível recorrer a soluções tecnológicas que permitam maior controle, como o ESPIM. É possível afirmar que a inclusão do sistema ESPIM às práticas pedagógicas em cursos de alfabetização e letramento digital para idosos é viável e eficaz em contextos educacionais. Além disso, há evidências de que pessoas idosas que são rotineiramente expostas a tecnologias móveis se sentem mais confortáveis e autoconfiantes usando a tecnologia (ERBES et al., 2014; ZHOU; RAU; SALVENDY, 2014). Assim, acreditamos que o uso de soluções de tecnológicas, como o "ajudante digital", para estimular a interação com dispositivos móveis rotineiramente pode proporcionar um impacto positivo no processo de alfabetização e letramento digital da população idosa.

Os resultados aqui relatados encorajaram nosso grupo de pesquisa a incluir formalmente o uso do sistema ESPIM como um recurso pedagógico nos cursos de alfabetização e letramento digital oferecidos desde a segunda metade do ano de 2017. Assim, o aplicativo atualmente está sendo usado desde o início dos cursos, totalizando cerca de 14 semanas de uso a cada oferta dos 
cursos. Estima-se que até o final de seis semestres de intervenções, cerca de 300 idosos tenham utilizado o aplicativo para incentivar o uso de dispositivos móveis fora do ambiente de sala de aula, auxiliando no objetivo de realizar a alfabetização e letramento digital efetivo dos idosos.

Vale ressaltar que o feedback dos participantes deste estudo sobre o aplicativo proporcionou que a interface do mesmo fosse aperfeiçoada e que fossem incorporadas novas funcionalidades com base nas sugestões feitas.

Por fim, agradecemos às agências de fomento pelo apoio financeiro: FAPESP (2015/18117-7; 2016/00351-6); (2017/19915-0); CNPq 312058/2015-2; CAPES (001).

\author{
A STUDY ABOUT ALPHABETIZATION \\ AND DIGITAL LITERACY FOR OLDER ADULTS \\ WITH THE SUPPORT OF A CUSTOMIZABLE \\ MOBILE APPLICATION
}

\section{abstract}

The use of computer systems for health care for older people is increasingly common. In the field of education, however, investigations focus more on technical issues of development or adaptation of technologies, rather than on the teaching-learning process and the applicability of using mobile devices. This work aimed to stimulate, monitor and evaluate the use of these devices in the natural environment by older participants in literacy and digital literacy courses. It also sought to promote new methodologies for alphabetization and digital literacy practices for older adults. A feasibility study was carried out exploring the use of a system called ESPIM, as a new educational resource in courses like this. ESPIM allows professionals from different domains to plan intervention programs to carry out data collections remotely with their populations of interest. This population answers questions programmed by professionals using a mobile application. Planned questions can be open questions, multiple choice, single choice, request to send media such as audio, video or images. In this qualitative study, for two consecutive weeks, participants received alerts and instructions, through this application, to carry out practical activities at home on the content learned in the classroom. The results obtained describe a positive behavior of the participants in terms of engagement in the execution of home activities, and also describe feedbacks about the application, such as facilities and difficulties, 
autonomy in the use and adherence to the technological resource as a way of supporting courses such as above citied.

keywords

Older Adults. Digital Alphabetization and Literacy. ESPIM System. Mobile Devices. Remote Interventions.

referências

BROOKE, John. SUS: a "quick and dirty" usability scale. In: JORDAN, Patrick; THOMAS, Bruce; MCCLELLAND, lan Lyall; WEERDMEESTER, Bernard (ed.). Usability evaluation in industry. London: Taylor \& Francis, 1996. p. 189-206.

CHEN, Kuanchin; CHEN, Jengchung Victor; YEN, David Chi-Chung. Dimensions of self-efficacy in the study of smart phone acceptance. Computer Standards and Interfaces, Netherlands, v. 33, n. 4, p. 422-431, 2011

CHIN, John; DIEHL, Virginia; NORMAN, Kent. Development of an instrument measuring user satisfaction of the human-computer interface. In: PROC. SIGCHI CONFERENCE ON HUMAN FACTORS IN COMPUTING SYSTEMS, 1988, New York. Anais [...]. New York: ACM CHli', 1988. p. 213-218.

CSIKSZENTMIHALYI, Mihaly; LARSON, Reed. Validity and reliability of the Experience-Sampling Method. The Journal of Nervous and Mental Disease, Baltimore, v. 175 ก. 9, p. 526-536, 1987

CUNHA, Bruna et al. Authoring of interventions with on-demand generation of screen for mobile devices. In: NETO, Manoel et al. (org.). Proceedings of the 24th Brazilian Symposium on Multimedia and the Web, WebMedia 2018, Salvador-BA, Brazil, October 16-19, 2018. [S.I.]: ACM, 2018. p. 145-149.

DAVIS, Fred. Perceived usefulness, perceived ease of use, and user acceptance of information technology. MIS Quarterly, Minneapolis, v. 13, n. 3, p. 319-340, Sep. 1989. DOl: $10.2307 / 249008$.

DEAR, Blake et al. Internet-delivered cognitive behavioral therapy for depression: a feasibility open trial for older adults. Australian and New Zealand Journal of Psychiatry, United Kingdom, v. 47, n. 2, p. 169-176, 2013.

ERBES, Christopher et al. Access, utilization, and interest in mHealth applications among veterans receiving outpatient care for PTSD. Military Medicine, United Kingdom, v. 179, n. 11, p. 1218-1222, 2014

HAUK, Nathalie; HÜFFMEIER, Joachim; KRUMM, Stefan. Ready to be a Silver Surfer? a meta-analysis on the relationship between chronological age and technology acceptance. Computers in Human Behavior, Netherlands, v. 84, p. 304-319, 2018.

JONES-KAVALIER, Barbara; FLANNIGAN, Suzanne. Connecting the digital dots: literacy of the 21st Century. Teacher Librarian, Canada, v. 35, n. 3, p. 13-16, 2008.

KUERBIS, Alexis et al. Older adults and mobile technology: factors that enhance and inhibit utilization in the context of behavioral health. Mental Health and Addiction Research, United Kingdom, v. 2, n. 2, p. 1-11, 2017.

LAUGWITZ, Bettina; HELD, Theo; SCHREPP, Martin. Construction and Evaluation of a User Experience Questionnaire. In: HOLZINGER, Andreas (org.). $\mathrm{HCl}$ and Usability for Education and Work. Berlin: Springer Berlin Heidelberg, 2008. p. 63-76. 
LEE, Jay; KAO, Hung-An; YANG, Shanhu. Service innovation and smart analytics for industry 4.0 and big data environment. Procedia College International pour la Recherche en Productique, Netherlands, v. 16, p. 3-8, 2014.

LÖCKENHOFF, Corinna; CARSTENSEN, Laura. Socioemotional selectivity theory, aging, and health: the increasingly delicate balance between regulating emotions and making tough choices. Journal of Personality, Farmington, v. 72, n. 6, p. 1395-1424, 2004.

MACHADO, Leticia Rocha; LONGHI, Magalí; BEHAR, Patricia Alejandra. SUS: domínio tecnológico: saberes e fazeres na educação a distância. In: BEHAR, Patricia Alejandra (ed.). Competências na Educação a Distância. Porto Alegre: Penso, 2013. p. 56-80,

PARK, Richard; MCLAUGHLIN, Anne. Designing displays for older adults. 1. ed. Boca Raton: CRC Press, 2010.

PIMENTEL, Maria et al. Enhancing older adults connectivity by introducing mobile devices communication tools. In: INTERNATIONAL CONFERENCE ON SOFTWARE DEVELOPMENT AND TECHNOLOGIES FOR ENHANCING ACCESSIBILITY AND FIGHTING INFO-EXCLUSION, 7., 2016, New York. Proceedings [...]. New York: Association for Computing Machinery, 2016. p. 353-361. DOl: 10.1145/3019943.3019994.

RODRIGUES, Kamila et al. ESPIM System: interface evolution to enable authoring and interaction with multimedia intervention programs. In: NETO, Manoel et al. (org.). Proceedings of the 24th Brazilian Symposium on Multimedia and the Web, WebMedia 2018, Salvador-BA, Brazil, October 16-19, 2018. [S.I.]: ACM, 2018. p. 125-132.

RUIZ, Jorge et al. Development and pilot testing of a self-management internet-based program for older adults with overactive bladder. Urology, United States, v. 78, n. 1, p. 48-53, 2011

TSAI, Hsin-Yi Sandy; SHILLAIR, Ruth; COTTEN, Shelia. Social support and "playing around": an examination of how older adults acquire digital literacy with tablet computers. Journal of Applied Gerontology, United States, v. 36, n. 1, p. 29-55, Sep. 2017.

VIEL, Caio et al. Personalized ubiquitous data collection and intervention as interactive multimedia documents. In: CAMILLERI, Kenneth; BONNICl, Alexandra (org.). Proceedings of the 2017 Symposium on Document Engineering, DocEng 2017, Valletta, Malta, September 4-7, 2017. [S.I.]: ACM, 2017. p. 223-226.

WEISER, Mark. The computer for the 21st century. Scientific American, United States, v. 265, n. 3, p. 223-226, 1991.

ZAINE, Isabela et al. An ubiquitous data collection and programmed intervention system using ESM and mobile devices. In: GOMES, Fábio et al. (org.). Proceedings of the 22nd Brazilian Symposium on Multimedia and the Web, Webmedia 2016, Teresina-PI, Brazil, November 8-11, 2016. [S.I.]: ACM, 2016. p. 13-14.

ZANDBERGEN, Rudd. Predicting persistency of usability problems based on error classification: a longitudinal study on improving mobility for the elderly. 2015. Thesis (Master's Degree) - Faculty of Behavioural, Management and Social Sciences, University of Twente, Enschede, 2015.

ZHOU, Jia; RAU, Pei-Luen Patrick; SALVENDY, Gavriel. Age-related difference in the use of mobile phones. Universal Access in the Information Society, Germany, v. 13, n. 4, p. 401-413, 2014. 